\title{
Iphigenie und ihre Mutter: Pragmatische Bemerkungen zur Iphigenie in Aulis
}

\author{
Giada Sorrentino
}

Einleitung: Interaktion und Charakterisierung in der Iphigenie in Aulis

Die Iphigenie in Aulis zeichnet sich durch die besondere Bedeutung aus, die im Plot den Schwierigkeiten und dem Scheitern der zwischenmenschlichen Kommunikation beigemessen wird. Die Handlung entwickelt sich um die Aufrechterhaltung und Enthüllung eines von Agamemnon gegen seine Familie und seine Tochter Iphigenie ersonnenen Trugs. Der Vater hat sie unter dem Vorwand, sie solle Achill heiraten, nach Aulis, wo er mit der griechischen Flotte nach Artemis' Willen festsitzt, kommen lassen. In Wirklichkeit soll sie geopfert werden, da dies die von der Göttin gestellte Bedingung ist, damit die Expedition beginnen kann. Der dauernde Widerstand einiger Figuren gegen die Opferung einerseits und andererseits die Meinungsumschwünge anderer erschweren die Entwicklung des Plots solange, bis das Mädchen durch seine Meinungsänderung selbst entscheidet, sich seinem Schicksal zu fügen.

In diesem dramatischen Gerüst liegt der Hauptgrund meiner Untersuchung des Dialogs in diesem Stück als Mittel zur Konstruktion und Gliederung des Plots sowie zur Charakterisierung der Figuren und ihrer Beziehungen. Die Pragmatik, die die Sprache als Form der Handlung betrachtet und sie mit den spezifischen Eigenschaften ihres Gebrauchskontexts in Beziehung setzt, kann einen grundlegenden Beitrag zu einer solchen Untersuchung leisten. Die Kategorien und Erklärungsmodelle, durch die sie den Gebrauch der Sprache in den kommunikativen Prozessen analysiert, haben sich seit langem auch als geeignete Instrumente erwiesen, um den aktionalen, interaktionalen und sozialen Aspekt der dramatischen Sprache zu erfassen. ${ }^{1}$ Daher verwende ich diese Instrumente - von der Sprechakttheorie über die Gesprächsanalyse bis zu den politeness-Studien - neben den traditionellen auf die Literatur angewandten Untersuchungsmethoden, um deutlicher hervorzuheben, wie die Figuren

1 Zur Anwendbarkeit der Pragmatik auf den Dialog des Dramas und zu ihren heuristischen Vorteilen vgl. u. a. Elam (2002: 142-167) und Hess-Lüttich (1980: 5-22, 2001: 1640-1655); van Emde Boas (2017) verwendet die verschiedenen Bereiche der Pragmatik in seiner Studie zur sprachlichen Charakterisierung der Figuren in Euripides' Elektra. 
durch ihr Interagieren die Handlung fortführen und gleichzeitig ihre dramatischen Rollen sowie ihre Beziehungen gestalten.

In diesem Beitrag beschränke ich mich darauf, einige Resultate meiner Untersuchung vorzustellen, die besonders die Charakterisierung der Figuren von Iphigenie und Klytaimestra im Dialog betreffen. Ich werde zeigen, wie die beiden Frauen im ganzen Stück hinsichtlich einiger grundlegender Aspekte ihres interaktionalen Verhaltens einander entgegengestellt werden und wie der Meinungsumschwung Iphigenies gegenüber der Opferung (ab Vers 1368) ihren Gegensatz nicht beendet, sondern unter umgekehrten Vorzeichen weiterführt und die Beziehung Mutter-Tochter im Schlussteil der Tragödie beeinflusst. ${ }^{2}$

\section{Selbstsichere Ehefrau, Mutter und Königin: die Charakterisierung Klytaimestras}

In den Gesprächen, an denen Klytaimestra beteiligt ist, zeigt sie interaktionale Verhaltensweisen, die trotz der Verschiedenheit der einzelnen Situationen bis zu Iphigenies Wandel konstant bleiben.

Ein erstes Kennzeichen betrifft die Art und Weise, wie sie das Wort ergreift bzw. übergibt. In der Mehrheit der Fälle wählt sie sich selbst als Sprecherin autonom aus, vor allem um Paarsequenzen zu eröffnen, also um Handlungen (wie z.B. Fragen) zu verwirklichen, die von den Gesprächspartnern bestimmte Reaktionen erwarten. ${ }^{3}$ Auf diese Weise gelingt es ihr, das Verhalten der Gesprächspartner zu beeinflussen und die Interaktion leicht in die von ihr gewünschte Richtung zu lenken.

Dies bemerkt man von ihrem ersten $\left(685^{-740}\right)$ bis zu ihrem letzten Gespräch vor dem Wandel Iphigenies (1345-1368). Im ersten folgen etwa 30 Verse lang

2 Der Text der Iphigenie in Aulis hat zahlreiche philologische Kontroversen ausgelöst, von denen viele immer noch offen sind: es lässt sich nämlich nicht bestreiten, dass das euripideische Grundgerüst verschiedenartige Änderungen erfahren hat, die es unmöglich machen, das ursprüngliche Werk von den Zusätzen zu unterscheiden. Ich folge der von Collard und Morwood (2017) besorgten Edition, welche die neueste ist und die meisten textkritischen bisher erschienenen Studien berücksichtigt. In den Anmerkungen werde ich nur auf die Fälle hinweisen, in denen ich von ihrem Text abweiche oder in denen sie selbst unsicher sind. Vgl. zu den wichtigsten Textproblemen außer Collard und Morwood (2017: 50-62) wenigstens die ausführlichen Darlegungen von Stockert (1992: 63-87).

3 Eine Paarsequenz (adjacency pair) ist die Abfolge von zwei Aussagen, die von verschiedenen Gesprächspartnern geäußert werden und so strukturiert sind, dass ein bestimmter erster Teil einen dazu passenden zweiten verlangt, z. B. Frage/Antwort, Gruß/Gegengruß usw. Vgl. Schegloff (2007:13-21). Die deutsche Terminologie folgt grundsätzlich Eberle (1997) und Meibauer $\left({ }^{2} 2001\right)$. 
Fragen der Königin an Agamemnon über von ihr gewählte Themen aufeinander, im zweiten wählt sie sich ständig selbst als Sprecherin aus, um ihren Gesprächspartner Achill zum Erzählen zu bringen, dessen Absichten zu erfahren und mit ihm zusammen den letzten Versuch zu unternehmen, Iphigenie zu retten.

Klytaimestras interaktionale Fähigkeit gewinnt im zweiten Dialog mit Agamemnon (1106-1145) an dramatischer Relevanz. Darin gelingt es der Königin, die inzwischen die wahren Absichten Agamemnons erfahren hat, in wenigen Zügen die Führungsrolle im Gespräch zu übernehmen und den Trug des Ehemanns zu entlarven. Der Dialog beginnt zwar auf Agamemnons Initiative, aber es ist Klytaimestra, die die verbale Auseinandersetzung gesucht hat, wie sie selbst in einem kurzen vorausgehenden Monolog (1098-1105) offenbart. Am Anfang zeigt sie sich fügsam und reagiert auf die Sprechhandlungen ihres Mannes, ohne zu zeigen, dass sie um seinen Plan weiß. ${ }^{4}$ Nachdem sie seinem Befehl, Iphigenie aus dem Zelt herausgehen zu lassen, gehorcht hat, zeigt sie ihre Fertigkeit, die aktive Rolle im Wortwechsel zu übernehmen. Zunächst erklärt sie sich zur einzigen Gesprächspartnerin des Ehemannes, indem sie sagt, dass sie für sich und für ihre Tochter sprechen wird (1121). Die von Agamemnon an das Mädchen gerichteten Fragen über die Gründe für seinen ausweichenden Blick und seine betrübte Miene rufen die folgenden Redebeiträge (turns) hervor:

[1] Euripides Iphigenie in Aulis 1124-1131
$\mathrm{K} \lambda . \varphi \varepsilon \hat{v}$.

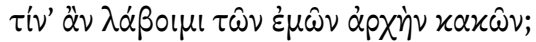

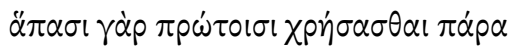

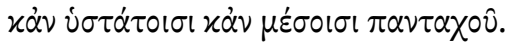

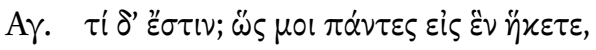

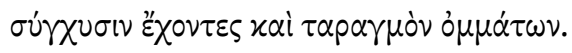

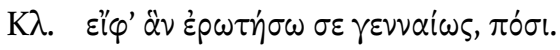

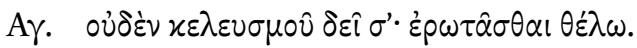

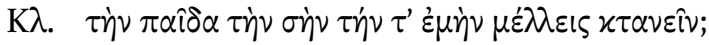

4 Der einzige Hinweis auf ihr Wissen, den Klytaimestra in dieser Passage des Dialogs gibt, ist

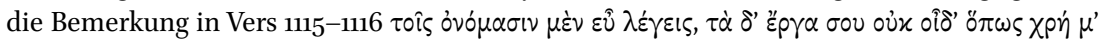

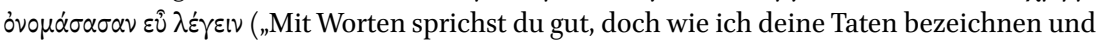
gut reden soll, das weiß ich nicht."). Diese wird aber durch das Wortspiel mit dem Ausdruck $\varepsilon \hat{}$ $\lambda \varepsilon^{\prime} \gamma \varepsilon เ v$, der sowohl, gut (wohl) reden' als auch ,das Richtige treffen` bedeutet, für Agamemnon zweideutig gemacht; vgl. Stockert (1992: 516). 
Kl. Ach, welchen Anfang meines Leidens soll ich wählen? Ein jedes kann ich ja als erstes nehmen, als letztes oder mittleres: Leid ist überall.

Ag. Was ist? Wie ihr beide doch einander gleicht: bedrückt erscheint ihr und mit verstörtem Blick.

Kl. Antworte ehrlich auf meine Frage, mein Gemahl.

Ag. Dazu brauchst du mich nicht aufzufordern: frage nur!

Kl. Hast du die Absicht, dein Kind und meines zu töten? ${ }^{5}$

Die Königin gibt zunächst eine allgemeine Antwort, in der sie über schwer zu beschreibende Übel spricht. Dies veranlasst Agamemnon, die Frage erneut zu stellen. Aber nicht einmal jetzt liefert sie eine klare Antwort, sondern fügt in die von Agamemnon eröffnete Frage-Antwort-Sequenz ein weiteres adjacency pair ein. Gemäß der Gesprächsanalyse eröffnet sie eine Einschubsequenz. ${ }^{6}$ In einer Aufforderung kündigt sie eine Frage ihrerseits an ihren Mann an. Diese Einschubsequenz bereitet also eine neue Paarsequenz vor, die nach Agamemnons Zustimmung von Klytaimestra eröffnet wird (1131 „Hast du die Absicht, dein Kind und meines zu töten?"). ${ }^{7}$ Durch diese konversationellen Züge gelingt es ihr, ihrem Mann die Rolle des Fragenden und damit die Zügel der Interaktion zu entreißen. Sie will, dass Agamemnon selbst auf seine Anfangsfrage antwortet und dabei seinen Plan offenbart. Ihre Anklage erscheint in Form einer Ja/Nein-Frage, die zwar schroff, aber weniger aggressiv als eine Anklage in assertiver Form ist. ${ }^{8}$ Dem Gesprächspartner würde eine einfache Verneinung genügen, um sie zurückzuweisen. Wegen seiner Unfähigkeit, eine solche zu liefern, wird ihn jedoch die Königin in den folgenden Versen (1132-1145) in die Enge treiben. Auf die Redebeiträge, mit denen der König versuchen wird, der Anklage auszuweichen, wird Klytaimestra mit ebenso vielen, in denen sie auf der Frage beharrt und das Fehlen einer Antwort immer signifikanter macht, reagieren. Von der so erreichten überlegenen Position aus wird sie schließlich explizit Agamemnons Spiel entlarven.

5 Die Übersetzung stammt, wo nicht anders angegeben, aus Blume (2014).

6 Einschubsequenzen (insertion sequences) sind Folgen von Redebeiträgen (etwa Paarsequenzen), die zwischen dem ersten und dem zweiten Teil einer umrahmenden Paarsequenz eingefügt werden und deren Vervollständigung derjenigen dieser vorausgeht. Vgl. Schegloff (2007: 97-114).

7 Die Paarsequenzen, die wie diese das Vorkommen einer folgenden vorankündigen, werden in der Gesprächsanalyse als Präsequenzen (pre-sequences) bezeichnet, vgl. dazu Schegloff (2007: 28-57).

8 Die Form der Frage kann die illokutionäre Kraft eines Sprechakts abmildern (Brown und Levinson ${ }^{2}$ 1987: 132-133). 
Die Sprechakte, welche Klytaimestra im Dialog verwirklicht, und ihre relative Frequenz in den verschiedenen Kontexten, in denen sie interagiert, bilden im Stück ebenfalls ein wirksames Instrument zu ihrer Charakterisierung. ${ }^{9}$

In dieser Hinsicht ist die erste Replik, die Klytaimestra bei der gemeinsamen Ankunft mit Iphigenie und dem kleinen Orest in Aulis ausspricht (6o7-63o), besonders interessant. Sie beginnt mit einem Dank an die Frauen des Chors, welche die königliche Familie willkommen geheißen haben, und entwickelt sich als lange Abfolge von direktiven Sprechakten (hauptsächlich Befehlen) ${ }^{10}$ an alle erreichbaren Anwesenden: begleitende Diener, die Frauen des Chors, Iphigenie und Orest. Die Zahl der Direktiva und ihrer Adressaten, ihre direkte Form (sie werden immer in Imperativsätzen geäußert), die unterschiedlichen Bereiche, die sie betreffen - vom Aussteigen der Sprecherin und ihrer Kinder aus dem Wagen über den Transport von Iphigenies Mitgift bis zur Versorgung der Pferde -, zeigen bereits bei Klytaimestras erstem Bühnenauftritt die Sicherheit, mit der sie die von ihren sozialen Rollen herrührende Autorität ausübt, und die Sorgfalt, mit der sie ihre Aufgaben erledigt. Diese werden sich in der Folge als die wichtigsten Merkmale ihres Charakters erweisen. ${ }^{11}$

Die in dieser Passage dominierenden Sprechakte nehmen auch sonst in Klytaimestras Sprechen bis zum Wandel Iphigenies eine wichtige Rolle ein. Die Königin macht reichlich von Direktiva Gebrauch, die sie an all ihre Gesprächspartner richtet, um ihre sozialen Rollen auszuüben und ihre Beziehungen zu gestalten. An Kinder und Untergebene richtet sie vor allem Befehle und Aufforderungen, die zu einer Mutter und Hausherrin passen. Gegenüber Achill äußert sie anfangs - schon in der Rolle der künftigen Schwiegermutter - Sprechakte des Vorschlags und der Aufforderung, nach der Entdeckung des unmittelbar bevorstehenden Opfers der Tochter dagegen eindringliche Bitten um Hilfe. ${ }^{12}$

Ich folge in der Regel der Einteilung der Sprechakte von Searle (1976). Die Fälle, in denen ich mich auf jüngere Studien stütze, die Searles Kategorien auf der theoretischen Ebene oder hinsichtlich der Anwendung auf reale bzw. literarische Interaktionen präzisiert oder korrigiert haben, werden jeweils in Anmerkung angegeben.

10 Gemäß Searle (1976:11) können die direktiven Sprechakte als „attempts (of varying degrees [...]) by the speaker to get the hearer to do something" definiert werden. Auf Risselada (1993: besonders 32-49) aufbauend, verfeinert und korrigiert teilweise Denizot (2011: 2324) Searles Kriterien der Einteilung. Sie liefert somit eine m.E. befriedigendere Klassifizierung der Unterarten der Direktiva, die den Akt der Frage ausschließt und Befehl/Aufforderung (injonction), Forderung/Bitte (requête), Flehen (prière), Vorschlag (proposition), Ratschlag (conseil), Anweisung (instruction) usw. klarer zu unterscheiden vermag.

11 Diesen von vielen für unecht oder suspekt gehaltenen, aber von anderen verteidigten Redebeitrag halten Collard und Morwood in ihrer Ausgabe mit Vorbehalt. Vgl. Collard und Morwood (2017: 401-402) und Stockert (1992: 380-381). 
Interessant ist, wie sich ihr Verhalten Agamemnon gegenüber ändert: Ihr Mann ist von Direktiva für eine bestimmte Dauer fast ganz ausgenommen, und zwar solange, bis Klytaimestra sich entschließt, seinen Plan zu entlarven. Von da an wendet sie sich an ihn immer häufiger mit diesen Sprechakten, die anzeigen, dass sie die Autorität des Gatten infrage stellt. ${ }^{13}$

Eine umfassende Untersuchung der von Klytaimestra verwirklichten Sprechakte zeigt ihre Vorliebe für Direktheit. Das gilt auch bezüglich der Sprechakte, welche einige politeness-Modelle als FTAs ( face threatening acts) bezeichnen, ${ }^{14}$ da sie das Bedürfnis nach Bestätigung sowie das Bedürfnis nach Freiheit, die das soziale Selbstbild ( face) eines Individuums darstellen, verletzen. ${ }^{15}$

Unter den politeness-Strategien, durch die Klytaimestra ihre FTAs abmildert, ist die Indirektheit selten und immer nur formal, da die FTAs aus dem Kontext und dem Kotext unmittelbar deutlich werden. ${ }^{16}$ Ein interessantes Beispiel begegnet vor der Entdeckung des Trugs und betrifft ihren FTA der Weigerung, dem Befehl Agamemnons, vor der Hochzeit Iphigenies nach Hause zu gehen, zu gehorchen:

\section{[2] Euripides Iphigenie in Aulis 733-740}

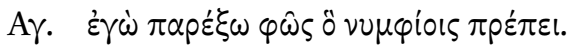

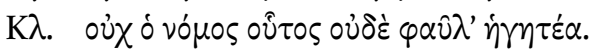

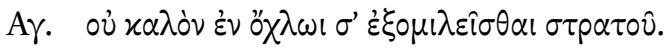

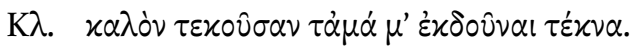

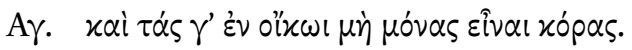

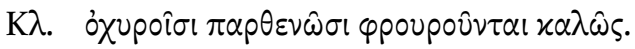

alten Diener $(867,872)$. Vor der Entdeckung des Truges wendet sie sich an Achill mit einer Aufforderung und einem Vorschlag (831-832), danach fleht sie ihn an (9oo-916, 985-989) und richtet wieder eine Bitte an ihn (995-996).

13 Die von Klytaimestra an Agamemnon gerichteten Direktiva sind eine Aufforderung (1129), die mehrmals wiederholt wird (1133 und 1135), ein Befehl in Form eines Rates (1143) und eine Bittrede (1146-1208). Der direktive Sprechakt in Vers 740 ist in Wirklichkeit Teil eines kommissiven Sprechakts der Ablehnung.

14 Das Modell, auf das ich mich hauptsächlich beziehe, ist das von Brown und Levinson $\left({ }^{2} 1987\right)$.

15 Vgl. Brown und Levinson (21987: 62-68) und Sektion B dieses Bandes.

16 Zur Tatsache, dass die Indirektheit (off-recordness) eines FTA nur formal ist, wenn der extralinguistische Kontext, der Kotext (d.h. die umgebenden Redebeiträge), sowie paralinguistische und nonverbale Hinweise nur eine einzige Interpretation dieses erlauben, vgl. Brown und Levinson ( ${ }^{2} 1987$ : 211-213). 


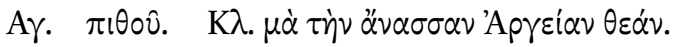

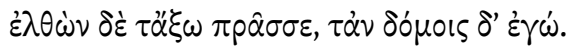

Ag. Ich werde das Licht scheinen lassen, das dem Brautpaar gebührt.

Kl. Das ist nicht der Brauch! Man darf dergleichen nicht geringschätzen.

Ag. Es ziemt sich nicht, dass du dich in der Masse des Heeres aufhältst.

Kl. Es ziemt sich aber, dass ich als Mutter mein Kind vermähle.

Ag. Aber auch, dass die Töchter zu Hause nicht allein bleiben.

Kl. In festen Mädchenzimmern leben sie wohlbehütet.

Ag. Gehorche!

Kl. Nein, bei der Göttin, die in Argos herrscht! Geh du und besorge die Dinge draußen; die im Haus besorge ich.

Agamemnon hat soeben Klytaimestra versichert, dass er auch die für die Feier von der Mutter zu verrichtenden Aufgaben erfüllen wird. Sie wendet ein, dass dieses Verhalten vom vópos (734) abweiche, der nicht unterschätzt werden dürfe. ${ }^{17} \mathrm{Ihr}$ Sprechakt ist zwar formal indirekt, wird aber in der kommunikativen Situation, in der er vorkommt, deutlich und löst einen Schlagabtausch über die Angemessenheit des Befehls aus. Auf die Bemerkung des Mannes, dass es nicht angebracht sei, dass Klytaimestra sich unter das Heer mische, antwortet sie in Vers 736, indem sie die Schicklichkeit der Anwesenheit der Mutter an der Hochzeit betont. Unmittelbar darauf (738) neutralisiert sie den Einwand des Gatten, dass das Verbleiben ihrer Töchter allein zu Hause unschicklich sei, durch die Versicherung, dass diese in Argos wohlbehütet sind. Trotz der formalen Indirektheit wird in diesen letzten Redebeiträgen ihre Ablehnung durch eine zweifache Wiederholung sogar verstärkt. Dient die exakte Wiederholung (736) des von ihrem Mann ausgesprochenen $x \alpha \lambda$ óv dazu, Klytaimestras Standpunkt demjenigen des Gatten deutlich entgegenzusetzen, schafft die variierte Selbstwiederholung mit $x \alpha \lambda \omega \hat{\omega}$ (738) eine Verbindung zum letzten eigenen Redebeitrag und verstärkt den darin verwirklichten Sprechakt. ${ }^{18}$

17 Zur politeness-Strategie des "give hints“, die im ersten Satz ihres Redebeitrags verwendet wird, um indirekt (off-record) einen FTA zu begehen, vgl. Brown und Levinson ( ${ }^{2} 1987$ : 213215). Der zweite Satz, der die Aufforderung, den vónoৎ nicht zu unterschätzen, enthält, macht den Sprechakt durch die passive Konstruktion mit dem Verbaladjektiv ohne die Erwähnung des Agens unpersönlich. Zu dieser Strategie vgl. Brown und Levinson ( ${ }^{2} 1987$ : 194-197).

18 Zur Wiederholung von Wörtern des Gesprächspartners (Fremdwiederholung), um Uneinigkeit auszudrücken, und zur Selbstwiederholung, um den eigenen Standpunkt zu verstärken, vgl. Bazzanella (1992: 438-439) und Locher (2004: 137-142). Zu den Formen und 
Diese Ausdrucksmodalitäten verdecken die illokutionäre Kraft des FTA der Weigerung nicht, sondern werden von Klytaimestra so lange verwendet, wie sie es für möglich hält, ihren Gatten durch die Begründung der Ablehnung seines Befehls umzustimmen. Zunächst erinnert sie Agamemnon an die Bedeutung des vómos, in der nachfolgenden Diskussion fordert sie gleiches Recht wie ihr Ehemann bei der Festsetzung dessen ein, was für sie und ihre Familie moralisch $x \alpha \lambda o ́ v$ ist. Damit verteidigt Klytaimestra ihre Rolle als Mutter und Hausherrin. Sobald sie begreift (739), dass es für den Meinungsaustausch keinen Raum mehr gibt, erwidert sie mit einer deutlichen Ablehnung, die durch die Schwurformel noch verstärkt wird, ${ }^{19}$ steckt dabei die Kompetenzsphären bei der Führung des oikos explizit ab und beendet autonom das Gespräch.

Klytaimestra mildert oft ihre FTAs durch die Erwähnung der Gründe, aus denen sie verwirklicht werden, ab. ${ }^{20}$ Diese werden in der Regel von ihr in den sozialen Rollen (gesellschaftliche Stellung, Geschlecht usw.), die sie oder ihre Gesprächspartner bekleiden, und in den Verhaltensweisen, die jeweils für sie vorgeschrieben sind, identifiziert. Außer den angeführten Beispielen sind diesbezüglich die Verse 903-908 besonders eindrücklich. Darin begründet die Königin ihre Bitte an Achill, das Leben Iphigenies zu retten, mit den Pflichten des Ehegatten, selbst wenn Achill, wie sie zugibt, nur zum Schein und ohne sein Wissen mit dieser Rolle bekleidet worden ist. ${ }^{21}$

Bevor sie merkt, dass sie betrogen worden ist (847), findet man hingegen sogar in den expressiven Sprechakten, ${ }^{22}$ die bisweilen kalt und formal erscheinen, kaum Hinweise auf ihre eigenen Gefühle. ${ }^{23}$ Bedeutsam ist der Gruß, den sie bei ihrer Ankunft an Agamemnon richtet:

Funktionen der Wiederaufnahme der vorhergehenden Redebeiträge im tragischen Dialog vgl. die Literaturangaben bei Bonifazi u. a. (2016: III.3.1.3).

Zu $\mu \alpha \dot{\alpha}$ mit Akkusativ als negative Erwiderung auf einen Imperativ (z. B. Ar. Thesm. 748) vgl. LSJ s.v. III.1.b (1070).

20 Zur Angabe der Gründe für einen FTA, um ihn abzumildern, vgl. Brown und Levinson (21987: 128).

21 Ein weiteres Beispiel begegnet in Vers 992-996: Bevor die Königin Achill explizit darum bittet, Iphigenies Leben zu retten, ohne dass das Mädchen das Zelt verlasse und ihn persönlich anflehe, unterstreicht sie (993), dass das Verhalten, welches sie ihrer Tochter ersparen will, sich für ein Mädchen nicht zieme. Zur Beachtung dessen, was angemessen ist, durch Klytaimestra vgl. Chong-Gossard (2008: 231-237).

Gemäß Searle (1976: 12) offenbaren die expressiven Sprechakte psychische Zustände, Einstellungen und persönliche Gefühle. Vgl. Risselada (1993: 4O-41) zur Schwierigkeit, diese und ihre Unterarten (z.B. Gruß, Glückwunsch, Dank, Entschuldigung, Beileid, je nach Kontext aber auch Wunsch, Ausruf usw.) zu definieren.

Die einzigen Hinweise darauf kommen in Vers 6o9, 691-692 und 844 vor. 
[3] Euripides Iphigenie in Aulis 633-634

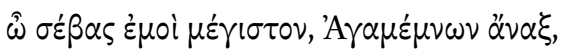

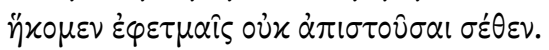

O höchste Majestät, Agamemnon, Herrscher! Nicht ungehorsam gegenüber deinen Befehlen sind wir gekommen. ${ }^{24}$

Die Königin wendet sich an ihren Gatten, den sie nach einiger Zeit wiedersieht, mit einer feierlich und kalt klingenden Hochachtung: Zuerst spricht sie ihn

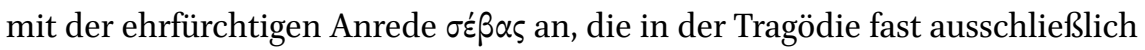
an Gottheiten gerichtet wird; ${ }^{25}$ die zweite Anrede mit dem Nominativ 'A $\gamma \alpha \mu \varepsilon$ $\mu \nu \omega \nu \alpha ٌ \nu \alpha \xi$ unterstreicht die Autorität des Ehemanns, aber nicht die Gefühle, die sie an ihren Gatten binden, was sonst in der Tragödie oft geschieht, wenn sich die Eheleute wiederbegegnen. ${ }^{26} \mathrm{Zu}$ den beiden Anreden passt die folgende Aussage gut, durch die Klytaimestra signalisiert, dass sie sich als gehorsame Ehefrau benommen hat.

Die beschriebenen interaktionalen Verhaltensweisen scheinen auf kohärente Weise dazu beizutragen, das Bild einer selbstsicheren und entschlossenen Figur zu entwerfen, die sich stark mit ihren gesellschaftlichen Rollen als Königin, Mutter und Ehefrau identifiziert. In ihrem Handeln spielen die sozialen Normen und Konventionen (im Gegensatz zu den Gefühlen) eine grundlegende Rolle. Diese Charakterisierung schafft einen scharfen Gegensatz zwischen Klytaimestra und Agamemnon, der sich vom Anfang an in jeder seiner

\footnotetext{
24 Übersetzung von mir.

25 Aesch. Cho. 157 ist der einzige sichere Passus einer Tragödie, in dem die Anrede an einen Menschen gerichtet wird, und zwar noch einmal an Agamemnon: In diesem Fall wendet

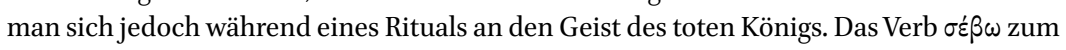
Ausdruck der Achtung innerhalb einer Ehe wird z. B. in Aesch. Ag. 925, Soph. oт 70o verwendet.

26 In der Tragödie richten Ehegatten Vokative und Ausdrücke der Zuneigung nicht nur bei unerwarteten Wiedererkennungen und Wiedervereinigungen aneinander - wie diejenigen in Eur. $H F$ 531, in dem Megara Herakles mit dem Vokativ $\hat{\omega} \varphi\left(\lambda \tau \alpha \tau^{\prime} \alpha \nu \delta \rho \hat{\omega} \nu\right.$, , liebster unter den Männern“ (531) grüßt, und Hel. 625 und 636, in denen die beiden Gatten, die sich endlich wiedererkannt haben, ähnliche Anreden aneinander richten -, sondern manchmal auch bei alltäglichen Treffen: so spricht z. B. in Eur. El. 345 die Protagonistin ihren Gatten mit dem Vokativ $\hat{\omega} \varphi\left(\lambda \tau \tau \alpha \tau^{\prime}(\varepsilon)\right.$ „o liebster“, an. Im Agamemnon wendet sich die aischyleische Klytaimestra, die nach dem Troianischen Krieg ihren Mann wiedersieht, mit

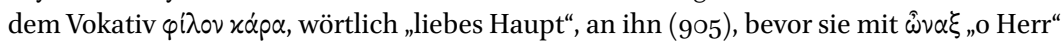
(907) den Ausdruck der Zuneigung mit demjenigen der Ehrerbietung verbindet.
} 
Rollen als inadäquat entpuppt. ${ }^{27} \mathrm{Im}$ folgenden werde ich zeigen, wie das kommunikative Verhalten der Königin auch in starkem Gegensatz zu demjenigen Iphigenies steht.

\section{Gefühle ausdrücken und austauschen: Iphigenies Kommunikation vor dem Wandel}

Einen ersten Einblick in die Unterschiede zwischen Tochter und Mutter geben schon die beiden über weite Strecken stichomythischen Dialoge, in denen zuerst Iphigenie (640-685) und dann Klytaimestra mit Agamemnon sprechen (685-740).

Der Dialog zwischen Iphigenie und Agamemnon ist der erste lange Wortwechsel, an dem das Mädchen teilnimmt. Dieser beginnt mit dem Austausch von Grüßen (640-643), fährt mit der Verwunderung Iphigenies über das Weinen des Vaters fort, das sie zu beenden versucht (644-650), entfaltet sich in der Folge um die Ankündigung Agamemnons einer Iphigenie bevorstehenden Reise (651-671), wird durch Hinweise des Königs über Opfer verlängert, die seiner Abreise von Aulis vorangehen werden (673-677), und endet damit, dass er der Tochter befiehlt, in sein Zelt zu gehen, und sich von ihr weinend verabschiedet $(678-685)$.

Die Unterschiede zwischen dem Verhalten Iphigenies und demjenigen Agamemnons in diesem Dialog hängen vor allem von ihrem Wissensgefälle bezüglich der Fakten ab. Iphigenie, die noch nichts über ihr künftiges Schicksal weiß, nimmt aktiv am Gespräch teil. Sie eröffnet es und führt es fort, indem sie fast immer autonom das Wort ergreift. Ihre Sprechakte sind immer explizit und klar und ihre nonverbale Kommunikation stimmt vollständig mit ihren Worten überein. ${ }^{28}$ Dagegen verhält sich Agamemnon eher reaktiv und nicht immer kollaborativ. Er begnügt sich in der Mehrzahl seiner Redebeiträge damit, der Tochter zu antworten, und erwidert auf ihre Fragen, die manchmal von ihm selbst eingeführte Themen betreffen, oft mit wenig klaren Antworten oder mit der Weigerung, eine Antwort zu geben. ${ }^{29}$ Seine Gesten stehen mehrmals mit

27 Zur Unzulänglichkeit Agamemnons in den verschiedenen Rollen, die er innehat, vgl. z.B. Michelakis (2006: 33-35) und die von ihm zitierte Sekundärliteratur.

28 Durch die vor dem Beginn des Dialogs (631-632) angekündigte Geste, dem Vater entgegenzulaufen und ihn zu umarmen, äußert Iphigenie ihren Enthusiasmus, ihn zu sehen, den sie darauf verbal ausdrücken wird (640-642). 
seinen Worten offenkundig in Widerstreit, so etwa, wenn er sagt, er sei glücklich, seine Tochter wiederzusehen, aber nicht aufhört zu weinen $\left(645^{-646}\right.$, 648-65o).

Andere konversationelle Verhaltensweisen Iphigenies verraten ihre Unreife in ihrem interaktionellen Gebrauch der Sprache. So bemerkt man etwa, dass sie nicht immer von ihrem Gesprächspartner Klarheit verlangt, was sonst in der Interaktion gewöhnlich geschieht. Manchmal geht sie nicht genauer auf den Sinn seiner dunklen Aussagen ein:

[4] Euripides Iphigenie in Aulis 658-66o

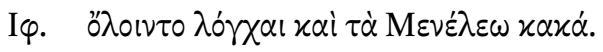

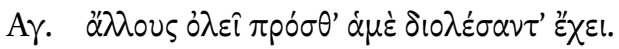

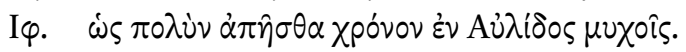

Iph. Verflucht seien die Waffen und das Unrecht an Menelaos!

Ag. Andere wird erst noch vernichten, was mich schon ganz vernichtet hat.

Iph. Wie lange schon bist du fort in der Bucht von Aulis!

Nachdem Iphigenie den Krieg und Menelaos' Übel verflucht hat, deutet Agamemnon ihr das traurige Schicksal an, das sie, die allgemein mit ä $\lambda_{0}$ ov bezeichnet wird, erwartet. Eine Frage Iphigenies würde genügen, damit die Wahrheit ans Tageslicht käme, aber sie stellt keine Forderung an den König, das Gesagte zu klären. Stattdessen knüpft sie an ihren letzten Redebeitrag an, um sich über die Länge seines Aufenthalts in Aulis zu wundern. ${ }^{30}$ In anderen Fällen akzeptiert sie seine Weigerung, auf ihre Fragen zu antworten. ${ }^{31}$ Dagegen vertieft sie mit weiteren Fragen alles, was der Vater über seine Gefühle und die sie beide betreffenden Angelegenheiten sagt. ${ }^{32}$

Dem Mädchen kommt es nicht darauf an, alles, was der Vater sagt, genau zu begreifen. Es beschränkt sich vielmehr darauf, über die Themen zu kommunizieren, in die es persönlich involviert ist, während es die Probleme übergeht,

hin- und herschwankt zwischen dem Wunsch zu sprechen und dem Unvermögen, diesen Wunsch zu realisieren" (Schwinge 1968: 189).

30 Zu Vers 659 sagt Schwinge (1968: 188): „Nur eine darauf bezogene Frage, und Agamemnon sähe sich durch Iphigenie selbst dazu aufgefordert, die Wahrheit auszusprechen. Doch Iphigenie überhört diesen Hinweis“. Dasselbe Verhalten registriert man einige Verse danach (661-662).

31 Das geschieht nach Vers 671 und 677-685.

32 Das passiert in Vers 644, 648-65o, 668-67o, 675-676. 
mit denen er sich als König und Feldherr beschäftigt. ${ }^{33}$ Iphigenie selbst bestätigt in diesem Dialog mit einem expliziten Hinweis auf die Verwendung der Sprache ihre Vorstellung von der Kommunikation. Nachdem ihr Vater gesagt

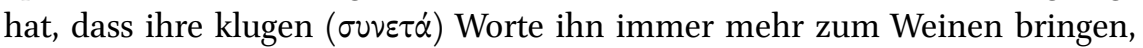

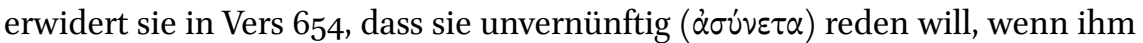
dies Freude bereiten $(\varepsilon \dot{\jmath} \varphi \rho \alpha v \hat{\omega})$ kann. Die Behauptung ist eine klare Äußerung der Zuneigung zum Vater. Zugleich zeigt Iphigenies geringe Beachtung rationaler Argumente, dass sie die verbale Kommunikation vorwiegend als Instrument auffasst, um Gefühle auszudrücken und hervorzurufen.

Die hohe emotionale Spannung ihrer Kommunikation wird von der hohen Zahl expressiver Sprechakte, die sie im gesamten Verlauf des Dialogs verwirklicht, bezeugt.

[5] Euripides Iphigenie in Aulis 640, 642, 644, 664, 666, 674

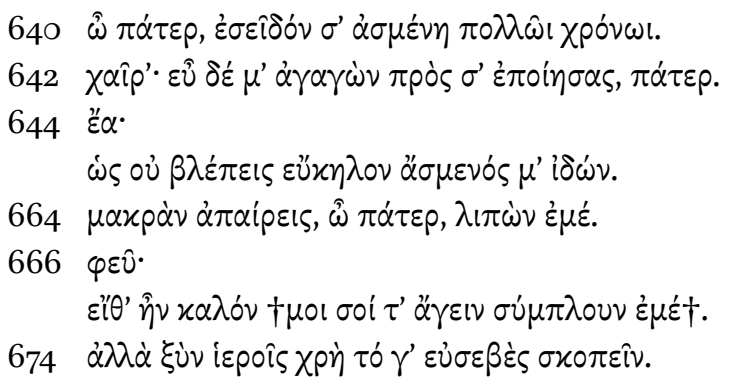

640 Vater, ich sehe dich mit Freuden nach langer Zeit.

642 Sei gegrüßt, wie gut, dass du mich herbeigerufen hast, Vater.

644 Ach, du blickst unruhig, und hast mich doch froh gegrüßt.

664 Auf eine weite Fahrt begibst du dich und lässt mich zurück.

666 Ach! Dürftest †du mich doch mitnehmen auf der Fahrt $\uparrow^{34}$

674 Ja, mit Hilfe heiliger Riten muss man auf Frömmigkeit achten.

Wie die zitierten Verse zeigen, gehören die von Iphigenie ausgedrückten expressiven Sprechakte den verschiedensten Untergattungen an: Gruß, Dank,

33 Iphigenies Verhaltensweisen zeigen, wie von Mellert-Hoffmann (1969: 68) beobachtet, „ihre Liebe zum Vater“, aber auch „ihre rührende Kindheit“, die sie dazu verleiten, die Dinge „nur von der persönlichen Seite, insofern diese Auswirkungen auf ihr Verhältnis zum Vater haben“, zu sehen.

34 Die cruces sind entsprechend dem gewählten griechischen Text von mir in die Übersetzung eingefügt worden. 
Ausdruck des Staunens, des Leidens, der Sehnsucht und der Zustimmung. In der vorangehenden Passage [4] kommen auch eine Verfluchung und ein weiterer Ausdruck der Sehnsucht vor.

Sowohl die angeführten als auch die übrigen Sprechakte Iphigenies sind auf der formalen Ebene voll von Zeichen des Affekts: Zahlreiche werden als Ausrufe ausgedrückt, ${ }^{35}$ bisweilen gehen ihnen verstärkende Interjektionen voraus ( $\left.{ }^{\prime \prime} \alpha, \varphi \varepsilon \hat{)}\right),{ }^{36}$ die Häufigkeit des Vokativs $(\hat{\omega}) \pi \dot{\alpha} \tau \varepsilon \rho$ bekräftigt den Kontakt zum Gesprächspartner, ebenso kommen gehäuft Personalpronomina der 1. und 2.

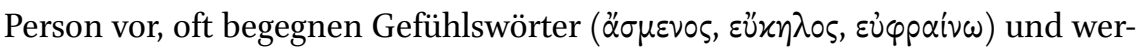
tende Begriffe ( $\varepsilon \hat{v}, x \alpha \lambda o ́ s) .{ }^{37}$

Ein nicht besonders reifes kommunikatives Verhalten, da in höchstem Maß auf den Ausdruck und Austausch von Emotionen und Gefühlen innerhalb einer Beziehung ausgerichtet, wird Iphigenie bis zu ihrem Wandel charakterisieren. Dieses unterscheidet Iphigenie nicht nur und nicht so sehr von Agamemnon, der in der Interaktion auf den Ausdruck seiner Gefühle nicht verzichtet, sondern auch und vielmehr von Klytaimestra.

Der Kontrast zwischen dem kommunikativen Verhalten der Tochter und demjenigen der Mutter zeichnet sich deutlich in der unmittelbaren Fortsetzung des Epeisodion mit dem schon erwähnten Dialog zwischen Klytaimestra und Agamemnon (691-740) ab. ${ }^{38}$ Darin treten die Konkretheit und die Rationalität Klytaimestras, die sich schon in ihrer ersten Replik (6o7-63o) gezeigt haben, schärfer zutage, da sie nur sachliche Themen wählt, und resultieren in einem Verhalten von Kälte gegenüber dem Gesprächspartner, an den nach dem Gruß (siehe [3]) nicht einmal eine Anrede gerichtet wird. Dazu kommen die umsichtige und gewissenhafte Aufmerksamkeit Klytaimestras in ihren Fragen und ihre Selbständigkeit in der Gesprächsführung (siehe [2]), die in klarem Gegensatz zur Naivität und Nachgiebigkeit der Tochter stehen.

35 Gemäß Risselada (1993: 41) nehmen die Ausrufe eine Mittelposition zwischen expressiven und assertiven Sprechakten ein, da sie „involve the expression of an emotion about a state of affairs whose existence is presupposed" und zugleich "describe this state of affairs".

36 Zur Funktion von है $\alpha$ in Vers 644 und von $\varphi \varepsilon \hat{~ i n ~} 666$ vgl. Biraud (2010: 47 bzw. 110).

37 Der Vokativ kommt in acht der 18 von Iphigenie geäußerten Repliken vor (640, 642, 662, 664, 656, 670, 672, 676). Gemäß Biraud (2010: 98) verstärkt in diesem Stück das häufige Vorkommen von $\hat{\omega}$ vor dem Vokativ die Nähe zum Gesprächspartner. Die Personalpronomina fehlen in sieben Repliken Iphigenies $(648,658,66 \circ, 662,668,674,676)$. $\alpha \sigma \mu \varepsilon v o \varsigma$ kommt in

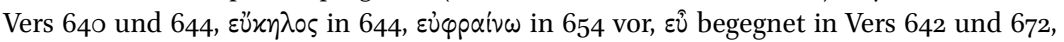

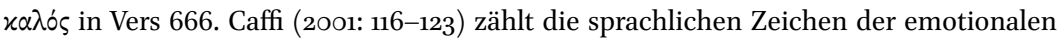
Beteiligung in der realen Kommunikation auf.

$38 \quad$ S. Abschnitt 2. 
Nach diesen beiden Stichomythien unterstreichen zwei ebenfalls nebeneinandergestellte rheseis im vierten Epeisodion die kommunikative Besonderheit Iphigenies und damit den Gegensatz zu Klytaimestra. Jede der beiden Frauen wendet sich an Agamemnon, um ihn zu bitten, auf die Opferung zu verzichten. Der Gegensatz zeichnet sich noch deutlicher ab, da beide Reden einen direktiven Sprechakt mit demselben perlokutionären Ziel verwirklichen und an denselben Adressaten innerhalb des gleichen Kontextes gerichtet sind. ${ }^{39}$ Eine komplexe Analyse der ganzen Reden nicht nur aus der pragmatischen, sondern auch aus der stilistischen und rhetorischen Perspektive, wäre wichtig, um alle Aspekte angemessen zu bewerten. ${ }^{40}$ Ich beschränke mich hingegen auf einige Bemerkungen, um das hervorzuheben, was am meisten die Kommunikation Iphigenies von derjenigen ihrer Mutter unterscheidet.

Dieses Mal spricht Klytaimestra als erste (1146-1208). Wie gewohnt behandelt sie der Reihe nach die Argumente, die sie für nützlich hält, um ihren Mann zu überreden, und offenbart dabei großes rhetorisches Geschick. ${ }^{41}$ Der Stil ihrer Rede ist oft emotionserregend, ohne dass sie selbst jemals ihren Gefühlen nachgibt. Von den rhetorischen Fragen über die sarkastischen Ausrufe bis zur Verwendung der oratio recta in imaginierten Situationen und zur Anrufung der Götter dient jedes Mittel dazu, das Unrecht und die Sinnlosigkeit der Tötung der Tochter zu belegen. ${ }^{42}$

Wenige Verse später beginnt Iphigenies Rede (1211-1252): Sie kündigt an, dass sie sich den Tränen als ihrer einzigen Fertigkeit anvertrauen wird. ${ }^{43}$ Von Anfang an thematisiert sie die eigene Unerfahrenheit im persuasiven Gebrauch des Wortes.

39 Beide Reden sind an Agamemnon gerichtete Bitten, auf das Opfer zu verzichten. Sie können also als direktive Makrosprechakte bezeichnet werden. Unter Makrosprechakt versteht man einen Text, der zwar aus mehreren Sprechakten bestehen kann, dessen kommunikative Hauptfunktion aber ein bestimmter Sprechakt bildet. Das Konzept von Makrosprechakt wurde von van Dijk (1977: 238-247) entwickelt.

40 Zur Bedeutung einer komplexen linguistischen und rhetorischen Methode zur Untersuchung der tragischen rheseis vgl. van Emde Boas (2017:47-50). Eine integrierte Perspektive zu deren Untersuchung ist indessen noch nicht vollständig entwickelt worden.

41 Aretz (1999: 165-169) liefert eine vertiefte rhetorische Analyse der Rede. Vgl. auch Gibert (2005: 230).

42 Rhetorische Fragen begegnen in Vers 1167, 1171-1178, 1185, 1186, 1187, 1188, 1191, 1192-1193, 1194-1195. Ein sarkastischer Ausruf erscheint in Vers 1168-1169. Die Verwendung der oratio recta in fingierten Situationen kommt in Vers 1177-1178 und 1197-1198 vor. Die Anrufung der Götter, die auf emphatisches $\mu \grave{\eta} \delta \hat{\eta} \tau \alpha$ („nein“, vgl. Denniston ${ }^{2} 1954: 276$ und Bonifazi u. a. 2016: III.3 § 49) folgt, erscheint in Vers 1183-1184.

43 Zum rhetorischen Gebrauch der Tränen in der antiken Literatur vgl. etwa Hagen (2016) und die von ihr angegebene Bibliographie sowie Calabrese in diesem Band. 
Tatsächlich entwickelt Iphigenie keine durchgehende Argumentation. Sie beschränkt sich auf die Bemerkung, dass es besser ist, (selbst unehrenhaft) zu leben, als zu sterben (1218-1219 und 1249-1250); außerdem stellt sie weinend nur einige erschrockene Fragen, was Helenas und Alexandros' Liebesaffäre mit ihr zu tun hat (1236-1237), und fleht Agamemnon im Namen seiner Vorfahren und ihrer Mutter Klytaimestra an. Sie bevorzugt die ihr vertrauten kommunikativen Modalitäten, wie der Kern ihrer Rede verdeutlicht:

[6] Euripides Iphigenie in Aulis 1220-1230

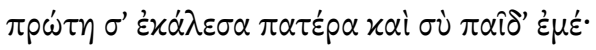

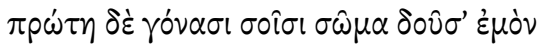

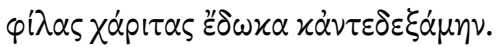

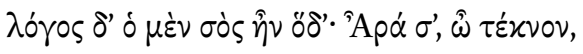

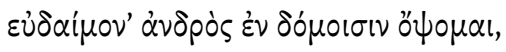

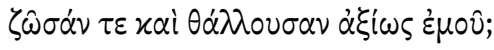

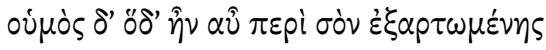

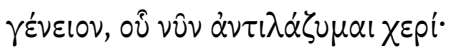

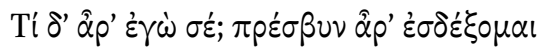

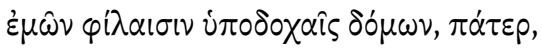

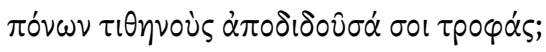

Als erste rief ich dich,Vater', und du mich ,Kind', als erste setzte ich mich auf deine Knie und streichelte und küsste dich und liess mich küssen. Da sprachst du so: „Werde ich dich, mein Kind, glücklich im Hause deines Ehemannes sehen, lebensfroh und blühend, meiner würdig?" Und ich erwiderte, während ich deinen Bart gefasst hielt, nach dem ich auch jetzt greife mit meiner Hand: „Und werde ich dich also, wenn du alt bist, aufnehmen und liebevoll in meinem Haus beherbergen, Vater, als Entgelt für die Mühen, dass du mich aufgezogen hast?“

Iphigenie versucht, ihre Bitte wirksam zu machen, indem sie dem Vater die gegenseitige Zuneigung, die sie seit jeher verbindet, in Erinnerung ruft. ${ }^{44}$ Sie beschränkt sich nicht darauf, die verbalen und nicht-verbalen Handlungen,

44 Die Erwähnung der Zuneigung, die Vater und Tochter seit jeher verbunden hat, ist ein Mittel, um die Reziprozität in den Gefühlen und die Gemeinsamkeit der Ziele auch in der Gegenwart zu verlangen. Zum Verlangen von Gegenseitigkeit und Kooperation als politeness-Strategie bei Forderungen und anderen FTAs vgl. Brown und Levinson ( ${ }^{2} 1987$ : 125-129). 
die sie mit dem Vater auszutauschen pflegte, erneut zu erwähnen, sondern vergleicht sie mit denjenigen, die sie in diesem Augenblick verwirklicht: Die emotionsgeladenen Anreden an den Vater, die Gesten, ihren Körper zum Vater hin zu bewegen und seine Knie und sein Kinn zu berühren - alles Elemente, die zum Ritual des Flehens (Hikesie) gehören - sind zum großen Teil dieselben, die sie in der Vergangenheit nur in der Absicht, ihre Zuneigung zum Vater zu zeigen, eingesetzt hat. Anders formuliert: Die üblichen Worte und Gesten, die sie an den Vater richtete und die plötzlich in die Vergangenheit gerutscht waren, leben jetzt wieder auf und füllen sich mit neuer illokutionärer Kraft auf. Handlungen, die vorher nur expressiv waren (Ausdrücke der Zuneigung), werden zu einem integrierenden Bestandteil des aktuellen, ritualisierten direktiven Sprechakts. Sind diese in der Vergangenheit vom Vater auf angemessene Art angenommen und erwidert worden, so erfordern sie auch jetzt, dass der Vater sie annimmt und auf die von der Tochter gewünschte Weise darauf reagiert.

Dies könnte einen Weg darstellen, um den rechten Ton gegenüber dem Vater zu treffen. ${ }^{45}$ Indessen wird die Sprecherin bald von ihren Gefühlen überwältigt. Die andauernde Unbeweglichkeit des Vaters, der sogar den Blick von ihr abwendet, veranlasst sie einige Verse später, vorübergehend das perlokutionäre Ziel der Rede aus den Augen zu verlieren und folgende Bitte zu formulieren:

[7] Euripides Iphigenie in Aulis 1238-1240

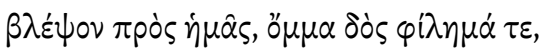

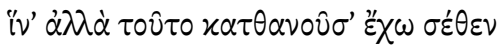

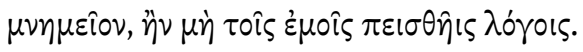

Schau mich an, schenk mir deinen Blick und einen Kuss, damit ich sterbend wenigstens dies als Erinnerung an dich behalte, wenn du dich nicht durch meine Worte bewegen lässt.

Durch Iphigenies Bitte an den Vater, sie anzublicken und ihr einen Kuss zu geben, selbst wenn er sich nicht durch ihre Worte umstimmen lasse, scheitert

45 Die Gegenseitigkeit des vergangenen verbalen und nicht-verbalen Verhaltens wird von Iphigenie wirksam durch das Nebeneinanderstellen von Verben des Gebens und Erhaltens (1222), durch den Chiasmus und den Parallelismus in der syntaktischen Struktur einiger Sätze (wie z.B. 1220) und durch das Zitieren der expressiven Sprechakte, die sie und ihr Vater austauschten, in oratio recta (1223-1225 bzw. 1228-1230) ausgedrückt. Vgl. dazu Aretz (1999: 169-173). 
der strategische Gebrauch der affektiven Kommunikation. Er weicht der Sehnsucht, diese wie in der Vergangenheit als Ausdruck gegenseitiger Zuneigung wieder zu erleben, und schwächt schließlich den Sprechakt des Flehens (ix£$\tau \varepsilon \dot{\varepsilon} \varepsilon(\nu)$ ab, anstatt ihn wirksamer zu machen.

Die Einbeziehung des kleinen Bruders Orest in die Bitte stellt Iphigenies letzten Versuch dar, die affektive Kommunikation zu verwenden, um der Rede Wirksamkeit zu verleihen: Sie bedient sich eines stummen Bittstellers als Gehilfen, den sie bittet, gemeinsam mit ihr zu weinen. Doch geschieht dies nicht, da er ihre Worte nicht versteht (1241-1248).

Die tiefgreifenden Unterschiede zwischen Iphigenie und der Mutter erscheinen vor allem in der Kommunikation mit Dritten (insbesondere mit Agamemnon). Die direkte Kommunikation zwischen den beiden Frauen auf der Bühne beschränkt sich bis zu Iphigenies Wandel auf kurze Wortwechsel, in denen Iphigenie ständig eine unterwürfige Position gegenüber ihrer Mutter einnimmt: Sie sucht Klytaimestras Zustimmung bezüglich des Verhaltens, das sie annehmen will (so in Vers 631-632, wenn sie zum Vater laufen will, um ihn zu umarmen), gehorcht ihren Anweisungen (so nach Vers 1343-1344, wenn sie auf die Aufforderung der Mutter vor Agamemnons Zelt bleibt), oder schließt sich einfach den Handlungen der Mutter an (so etwa, wenn sie in Vers 12791282 auf die Klage der Mutter mit einer Klage antwortet, die bezüglich der Töne, des Vokabulars und des Rhythmus jener ähnlich ist). ${ }^{46}$

Der Wandel Iphigenies hinsichtlich ihres Schicksals (ab Vers 1368) zeigt sich auch anhand einer tiefgreifenden und ebenso plötzlichen Änderung ihrer kommunikativen Modalitäten, der ein symmetrischer und entgegengesetzter Wandel bei Klytaimestra entspricht. Dadurch wird auch die Beziehung MutterTochter vor ihrer endgültigen Trennung beeinflusst.

\section{Iphigenies und Klytaimestras kommunikativer Wandel}

In der Rede in Vers 1368-1401 sind die wichtigsten Änderungen des kommunikativen Verhaltens Iphigenies sofort sichtbar. Darin verknüpfen sich eine ihr bisher unbekannte argumentative Fähigkeit, ein ebenso neues Vertrauen in die Wirksamkeit ihrer Worte und wichtige Änderungen auf der konversationellen und illokutionären Ebene derart miteinander, dass dadurch die Form und Struktur der Rede bestimmt werden.

46 Die Echtheit der Verse 1276-1282 ist sehr umstritten, vgl. Collard und Morwood (2017: 556). 
Hatte Iphigenie sich kurz zuvor einer Rhetorik der Tränen und Gefühle anvertraut und war Opfer derselben geworden, erscheint sie jetzt unverkrampft und entschlossen im Gebrauch des Wortes. Dies zeigt schon die Art und Weise, auf die Iphigenie das Wort ergreift, deren unerwarteter Charakter während einer Interaktion, an der sie nicht beteiligt war, von der $\alpha \dot{\nu} \tau i \lambda \alpha \beta \dot{\eta}$ unterstrichen wird. ${ }^{47}$ Im Laufe der Rede entwickelt sie verschiedene Argumente zugunsten ihrer plötzlichen Entscheidung, den Tod zu erleiden. Sie hält eine geordnete und gut gegliederte Rede, deren grundlegende Momente (den Beginn, die Ankündigung der eigenen Entscheidung, den Anfang des argumentativen Teils, den Übergang zu einem neuen Argument) sie durch explizite Hinweise auf die Worte, die sie ausspricht, unterteilt. Diese Hinweise haben meistens die Form von Aufforderungen an die Gesprächspartner (insbesondere an die Mutter), auf ihre Rede zu hören und deren Richtigkeit zu beachten. ${ }^{48}$ Ihre Funktion besteht darin, die in der Rede enthaltenen Sprechakte zu verstärken. Dabei dominiert die kommissive Illokution, ${ }^{49}$ da die Verpflichtung, den eigenen Körper zur Rettung Griechenlands herzugeben, im Mittelpunkt der Rede steht (z.B. 1383).Zahlreich sind die dazu komplementären direktiven Sprechakte, die nicht nur an ihre Mutter (1371-1373), ${ }^{50}$ sondern an ganz Griechenland (1398) und in dritter Person an Achill (1392-1393) gerichtet werden. Das Mädchen, welches sich kurz zuvor von der Mutter leiten ließ und in der Kommunikation expressive Sprechakte bevorzugte, lässt Illokutionen dominieren, die den Sprecher bzw. den Adressaten verpflichten, den Status der Dinge zu verändern. ${ }^{51}$

Im letzten Wortwechsel zwischen Iphigenie und Klytaimestra nach Achills Abgang von der Bühne (1433-1466) - der ersten und einzigen langen verbalen Auseinandersetzung zwischen den beiden in dieser Tragödie - wird eine totale Umkehrung der von ihnen in der Vergangenheit geführten kommunikativen

47 Der Beginn einer rhesis durch die Unterbrechung eines laufenden Dialogs mitten im Vers ist besonders signifikant, da er in der Tragödie selten und auf spezielle Situationen wie auf den unerwarteten Bühnenauftritt des neuen Sprechers beschränkt ist (so etwa in Vers 414 dieser Tragödie). Vgl. dazu Collard und Morwood (2017: 584-585), Aretz (1999: 19o).

48 Diese Hinweise findet man in Vers 1368-1369 (Redebeginn), 1374 (Ankündigung der Entscheidung), 1377 (Beginn des argumentativen Teils), 1392 (Übergang zu einem neuen Argument).

49 Searle (1976: 11) folgend, bezeichne ich diejenigen Sprechakte als Kommissiva, die den Sprecher auf die Ausführung einer zukünftigen Handlung verpflichten, wie z.B. das Versprechen oder die Drohung.

$5^{\circ}$ Im übrigen verschont Iphigenie ihre Mutter nicht mit Kritik (1369-1370).

$5^{1}$ Kommissiven und direktiven Sprechakten ist nach Searle (1976: 11-12) die Beziehung von Wort und Welt, die sie bewirken, gemeinsam: mit beiden Typologien von Sprechakten versucht man, die Welt an die eigenen Worte anzupassen und nicht umgekehrt. 
Verhaltensweisen offenbart. Schon die ersten Verse zeigen einen deutlichen Wandel in der Interaktion zwischen Mutter und Tochter:

[8] Euripides Iphigenie in Aulis 1433-1436

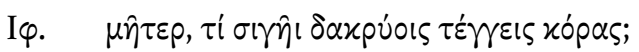

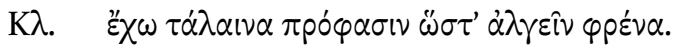

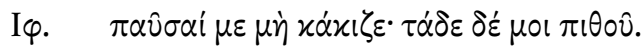

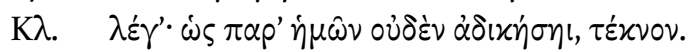

Iph. Mutter, warum bist du still und füllst deine Augen mit Tränen?

Kl. Ich Arme habe Grund, von Herzen betrübt zu sein.

Iph. Halt ein! Mach nicht weich und gewähre mir dies eine.

Kl. Nun, was? Von mir erfährst du kein Unrecht, Kind.

Der Anstoß, die Interaktion wiederaufzunehmen, geht vom Mädchen aus, das sich darüber wundert, dass die Mutter nach seiner Rede nicht mehr zu Wort gekommen ist, sondern schweigend weint. Nachdem Klytaimestra ihren $\mathrm{Zu}$ stand mit einer Klage begründet, antwortet Iphigenie auf unerwartete Weise: Mit drei aufeinanderfolgenden Imperativen fordert sie von der Mutter explizit und ohne jegliche Abmilderung, mit ihrem Verhalten, das sie feige macht, aufzuhören, und befiehlt ihr, auf das zu achten, was sie sagen wird. Die Mutter gibt ihr Einverständnis und überlässt ihr das Wort. ${ }^{52}$

Iphigenie dominiert das Gespräch vom Beginn bis zum Ende. Auch wenn sie die Mutter nicht daran hindert, autonom das Wort zu ergreifen, führt sie das erste Thema ein, bestimmt den Augenblick der Beendigung des Gesprächs (1458-1466) und zögert in seinem Verlauf nicht, die Regeln der Konversation zu verletzen, sooft die Mutter den Dingen einen anderen Lauf zu geben versucht (etwa durch die Unterbrechung des Redebeitrags der Mutter in Vers 1459 oder durch die Unterlassung des Abschiedsgrußes in 1464-1466).

Auf der illokutionären Ebene stellt man die hohe Zahl der direktiven Sprechakte in Iphigenies Sprechen fest. Auf das Verbot an die Mutter, nach ihrem Tod Trauerkleider zu tragen (1437-1438), folgen unter anderem Anordnungen, wie man ihren Leichnam zu ehren habe (1442, 1444), Forderungen betreffend die Familienmitglieder $(1448,1450,1454)$ und Anweisungen, mit denen Iphigenie ihren Abgang zum Ort des Opfers organisiert (1459-1461, 1466).

$5^{2}$ In Klytaimestras Redebeitrag dient m.E. der Satz „von mir erfährst du kein Unrecht“ als Beweis für die unerwartete Schroffheit von Iphigenies Befehlen. 
Die Direktiva werden direkt und explizit ausgedrückt (in den meisten Fällen mit Imperativ oder $\mu$ ' und Imperativ Präsens bzw. Konjunktiv Aorist). Auf diese folgt oft als Abmilderung die Erklärung ihrer Gründe, aber nicht unmittelbar, sondern in der Regel nach Einwänden der Mutter. Im Gegensatz zu früher

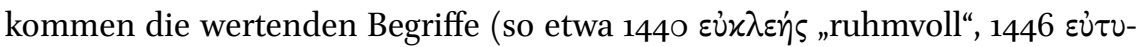

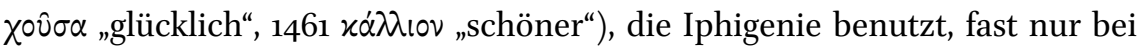
der Begründung ihrer Direktiva vor.

Der Bevorzugung der Direktiva entspricht eine Abneigung, verbale und nonverbale Gefühlsäußerungen zu verwirklichen. Diesbezüglich ist folgende Passage signifikant:

[9] Euripides Iphigenie in Aulis 1447-1452

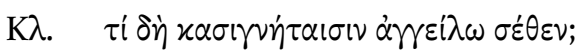

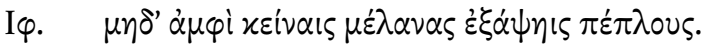

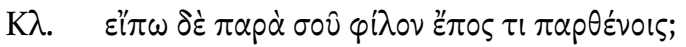

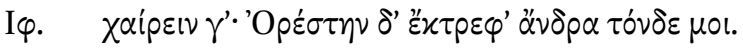

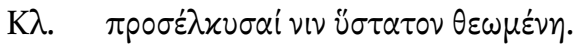

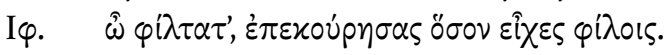

Kl. Was aber soll ich deinen Schwestern melden?

Iph. Auch sie kleide nicht in schwarze Gewänder.

$\mathrm{Kl}$. Soll ich von dir ein liebes Wort den Mädchen sagen?

Iph. Ein frohes Lebewohl. Und meinen Orest ziehe auf zum Mann.

Kl. Umarme ihn, denn du siehst ihn zu letzten Mal.

Iph. Du mein Liebster, du hast uns geholfen, so gut du es vermochtest.

Die Mutter, die das Wort vor allem dazu ergreift, um Iphigenie die Erfüllung einiger letzter Gefallen nach dem Tod anzubieten, fragt, was sie ihren Schwestern von ihr ausrichten soll. Iphigenie antwortet mit einem Befehl, wobei sie ein Verhalten annimmt, das ganz weit von demjenigen vor dem Wandel entfernt ist. Die Mutter muss im folgenden Redebeitrag ausdrücklich nach einem

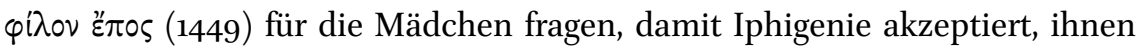
einen raschen Gruß zu senden, worauf sogleich wieder ein Befehl an die Mutter folgt. An den kleinen Orest richtet sie in Vers $145^{2}$ einen liebevollen Sprechakt des Dankes, aber auch in diesem Fall auf Klytaimestras Bitte. ${ }^{53}$

53 Die wenigen von Iphigenie gebrauchten Gefühlswörter werden in den expressiven Sprechakten an die Geschwister (z. B. $\hat{\omega} \varphi \hat{i} \lambda \tau \alpha \tau \varepsilon$ 1452) und in der Bitte an ihre Mutter, den Vater 
Ihr gegenüber zeigt das Mädchen einen wachsenden emotionalen Abstand. Dieser zeigt sich durch zahlreiche kommunikative Zeichen. Zunächst ist beachtenswert, dass Iphigenie nur zweimal - im Aufruf des bereits erwähnten ersten Redebeitrags (1433) und kurz vor ihrem Abgang (1460) - ein farbloses $\mu \hat{\eta} \tau \varepsilon \rho$ an die Mutter richtet, während diese den Vokativ $(\widehat{\hat{\omega}}) \tau \varepsilon \dot{\varepsilon}$ xvov doppelt so häufig $(1436,1439,1445,1464)$ gebraucht. Zum schon erwähnten Fehlen eines Abschiedsgrußes gesellt sich die Tatsache, dass Iphigenie sich weigert, das Weinen mit der Mutter zu teilen. Mit dem kalten und distanzierten Satz oủx $\dot{\varepsilon} \hat{\omega}$

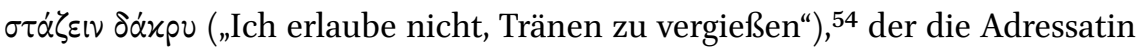
nicht einmal erwähnt, spricht sie sogar ein Verbot zu weinen aus und beendet damit das Gespräch (1466). ${ }^{55}$

Seitdem Iphigenie die Rolle der siegenden Heldin angenommen hat, bedient sie sich eines radikal veränderten kommunikativen Verhaltens und nimmt eine resolute, direkte und autoritäre Haltung ein. Sie ergreift entschlossen die Zügel des Lebens, das ihr bleibt, und emanzipiert sich vom Willen und der Kontrolle der Mutter. Von dieser verlangt sie die Annahme ihrer Entscheidungen sowie ihrer neuen Definition und Festsetzung der moralischen Werte, die ihr Handeln motivieren. ${ }^{56}$

Ihrem Wachstum entspricht eine Schwächung Klytaimestras. Die Königin erfährt den Entschluss Iphigenies als eine Aushöhlung ihrer Rolle als Mutter, da sie unfähig gewesen ist, ihre Tochter zu retten. Das Schweigen und das Weinen sind die kommunikativen Signale für ihren Gemütszustand. Sie kann nur noch ihre affektive Bindung zur Tochter ausdrücken. Aber Iphigenie weiß ihrerseits, dass sie auf ihre emotionalen Bindungen verzichten muss, um ihre Entschei-

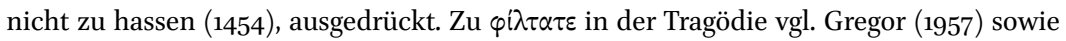
van Emde Boas (2017: 99), in der Prosa vgl. Dickey (1996: 119-120, 135-138).

54 Übersetzung von mir. Das Fehlen des Akkusativs des Adressaten macht den Befehl allgemeiner. Was sonst eine negative politeness-Strategie ist (vgl. Brown und Levinson ${ }^{2} 1987$ : 190), kann in einer so intimen und heiklen Interaktion wie dieser nur dazu dienen, die Sprecherin von der Adressatin zu distanzieren (vgl. Brown und Levinson 21987: 93).

55 Nach Ende des Gesprächs wendet sich das Mädchen noch einmal während der Schlussmonodie an seine Mutter, nur um zu bestätigen, dass es ihre Tränen nicht erwidern wird (1487-149o). Das Fehlen des Abschiedsgrußes und des gemeinsamen Weinens sticht besonders hervor, wenn man diese mit anderen analogen Situationen der euripideischen Tragödie vergleicht. Sowohl in Hec. $415^{-431}$ als auch in Heracl. 574-596 verzichten die Mädchen, die zum Sterben gehen, nicht darauf, den Gesprächspartnern spontan einen Abschiedsgruß zu geben und Grüße an ihre abwesenden Lieben auszurichten. In Hec. 433-434 steckt das Weinen der Protagonistin dasjenige ihrer Tochter und umgekehrt an. Zum „contagio delle lacrime“ als Zeichen einer affektiven Bindung zwischen zwei Figuren in der antiken Literatur vgl. Ricottilli (2000: 192-193).

Vgl. dazu Chong-Gossard (2008: 238-240). 
dung umsetzen zu können. Je mehr daher die Mutter versucht, sich der Tochter zu nähern, desto mehr entfernt sich diese bis hin zur definitiven, kalten Trennung.

Die Anwendung der Kategorien der Pragmatik auf den Dialog der Iphigenie in Aulis hat m.E. ein tiefer greifendes Verständnis und eine wirksamere Beschreibung des kommunikativen Verhaltens Iphigenies und Klytaimestras ermöglicht. Insbesondere hat sie erlaubt, die Kohärenz aufzuzeigen, mit der die Mutter und die Tochter hinsichtlich der Kommunikation sowohl vor als auch nach dem Wandel Iphigenies einander entgegengestellt werden. Ihr Gegensatz dient sicherlich dazu, die Einzigartigkeit der Reifung Iphigenies zu unterstreichen: Das zuvor naive und emotionale Mädchen ist nicht nur plötzlich erwachsen geworden, sondern hat sogar den Status einer Heldin erlangt, die imstande ist, die Schranken einer starken, aber konventionellen Frau wie ihrer Mutter zu überwinden.

Die Tatsache schließlich, dass die beiden Frauen sich zwar in vielen Gesichtspunkten unterscheiden, aber am Ende der Tragödie als die stärksten und entschlossensten Figuren hervorgehen, bildet einen weiteren Gegensatz zwischen ihnen und den männlichen Helden dieses Stücks, welche von Anfang an als wankelmütig und gleichzeitig starr, ehrgeizig und feige, gewalttätig und dennoch unfähig dargestellt werden. ${ }^{57}$

\section{Danksagung}

Dieser Beitrag stellt einige Resultate des Forschungsprojektes „Kommunikation, Handlung und Figuren in der Tragödie des Euripides" vor, das ich 20152017 an der Albert-Ludwigs-Universität Freiburg i. Br. durchgeführt habe. Die Fritz Thyssen Stiftung hat das Projekt finanziell unterstützt. Bernhard Zimmermann und David Konstan haben die Ergebnisse meiner Forschung gelesen und mir wertvolle Ratschläge erteilt. Severin Hof, Federica Iurescia und Gunther Martin haben mir mit wichtigen Anregungen bei der Gestaltung dieses Aufsatzes geholfen. Ihnen allen gebührt großer Dank.

57 Dass Iphigenie sich, wie Andò (2008) und Battezzato (2017: 173-174) gezeigt haben, mit ihrem Wandel die Werte zu eigen macht, die der politischen und patriotischen Sphäre angehören und somit für den männlichen Bereich typisch sind, macht m.E. die Unzulänglichkeit der männlichen Figuren, diese Werte zu verteidigen, nur noch deutlicher. 


\section{Bibliographie}

Andò, V., (2008), ,Un corpo di donna per fare la guerra. Lettura della Ifigenia in Aulide di Euripide', Storia delle donne 4, 71-82.

Aretz, S., (1999), Die Opferung der Iphigenia in Aulis: Die Rezeption des Mythos in antiken und modernen Dramen, Stuttgart.

Battezzato, L., (2017), ,Change of Mind, Persuasion, and the Emotions: Debates in Euripides from Medea to Iphigenia at Aulis', Lexis 35, 164-177.

Bazzanella, C., (1992), ,Aspetti pragmatici della ripetizione dialogica', in G. Gobber (Hg.), La linguistica pragmatica. Atti del XXIV Congresso della SLI, Rom, 433-454.

Biraud, M., (2010), Les interjections du théâtre grec antique. Étude sémantique et pragmatique, Louvain-la-Neuve.

Blume, H.-D., (2014), Euripides, Iphigenie in Aulis, Stuttgart.

Bonifazi, A., Drummen, A. und Kreij, M. de, (2016), Particles in Ancient Greek Discourse: Five Volumes Exploring Particle Use Across Genres, Washington, D.C. https:// chs.harvard.edu/CHS/article/display/6391.particles-in-ancient-greek-discourse [25/06/2019].

Brown, P. und Levinson, S.C., ( ${ }^{2} 1987$ [1978]), Politeness: some Universals in Language Usage, Cambridge.

Caffi., C., (2001), La mitigazione: un approccio pragmatico alla mitigazione nei contesti terapeutici, Münster.

Chong-Gossard, J.H.K.O., (2008), Gender and Communication in Euripides' Plays: between Song and Silence, Leiden.

Collard, C. und Morwood, J., (2017), Euripides, Iphigenia at Aulis, 2 Bände, Liverpool.

Denizot, C., (2011), Donner des ordres en grec ancien: étude linguistique des formes de l'injonction, Mont-Saint-Aignan.

Denniston, J.D., ( ${ }^{2} 1954$ [1933]), The Greek Particles, Oxford.

Dickey, E., (1996), Greek Forms of Address: from Herodotus to Lucian, Oxford.

Dijk, T.A. van, (1977), Text and Context: Explorations in the Semantics and Pragmatics of Discourse, London.

Eberle, T.S., (1997), ,Ethnomethodologische Konversationsanalyse‘, in R. Hitzler und A. Honer (Hg.), Sozialwissenschaftliche Hermeneutik, Opladen, 245-279.

Elam, K. (22002 [1980]), The Semiotics of Theatre and Drama, London/New York.

Emde Boas, E. van, (2017), Language and Character in Euripides' Electra, Oxford.

Gibert, J., (2005), ,Clytemnestra's First Marriage: Euripides' Iphigenia in Aulis', in V. Pedrick und S.V. Oberhelman (Hg.), The Soul of Tragedy: Essays on Athenian Drama, Chicago/London, 227-248.

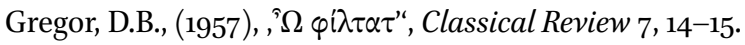

Hagen, J., (2016), ,Emotions in Roman Historiography: the Rhetorical Use of Tears as a Means of Persuasion', in E. Sanders und M. Johncock (Hg.), Emotion and Persuasion in Classical Antiquity, Stuttgart, 199-212. 
Hess-Lüttich, E.W.B., (1980), ,Literatur und Konversation. Der literarische Dialog als Gegenstand empirischer Textwissenschaft', in E.W.B. Hess-Lüttich (Hg.), Literatur und Konversation, Wiesbaden, $5^{-22}$.

Hess-Lüttich, E.W.B., (2001), ,Gesprächsanalyse in der Literaturwissenschaft', in K. Brinker, G. Antos, W. Heinemann und S.F. Sager (Hg.): Text- und Gesprächslinguistik / Linguistics of Text and Conversation, Teilband 2, Berlin/New York, 1640-1655.

Locher, M.A., (2004), Power and Politeness in Action: Disagreements in Oral Communication, Berlin/New York.

Meibauer, J., (22001 [1999]), Pragmatik. Eine Einführung, Tübingen.

Mellert-Hoffmann, G., (1969), Untersuchungen zur „Iphigenie in Aulis“ des Euripides, Heidelberg.

Michelakis, P., (2006), Euripides, Iphigenia at Aulis, London.

Ricottilli, L., (2000), Gesto e parola nell'Eneide, Bologna.

Risselada, R., (1993), Imperatives and other Directive Expressions in Latin: a Study in the Pragmatics of a Dead Language, Amsterdam.

Schegloff., E.A., (2007), Sequence Organization in Interaction, Cambridge.

Schwinge, E.-R., (1968), Die Verwendung der Stichomythie in den Dramen des Euripides, Heidelberg.

Searle, J.R., (1976), ,A Classification of Illocutionary Acts', Language in Society 5, 1-24.

Stockert, W., (1992) Euripides, Iphigenie in Aulis, 2 Bände, Wien. 